\title{
Complex tibial fractures are associated with lower social classes and predict early exit from employment and worse patient-reported QOL: a prospective observational study of 46 complex tibial fractures treated with a ring fixator
}

\author{
Rasmus Elsoe $^{1} \cdot$ Peter Larsen $^{2} \cdot$ Juozas Petruskevicius $^{1} \cdot$ Søren Kold ${ }^{1}$
}

Received: 10 January 2017 / Accepted: 29 October 2017 / Published online: 4 November 2017

(C) The Author(s) 2017. This article is an open access publication

\begin{abstract}
The long-term outcomes following complex fractures of the tibia are reported to carry a risk of knee pain, malalignment, articular injury and post-traumatic osteoarthritis. The main objective of this study was to account for the patient-reported quality of life (QOL) 12 months after ring fixator removal in patients with a complex tibial fracture. Secondary objectives included a review of the socioeconomic characteristics of the patient group and the rate of return to work in the study period. A prospective follow-up study was conducted of 60 patients with complex fractures of the tibia treated with ring external fixation. Patient-reported outcomes, radiological outcomes and socio-economic status including employment status of the patients were obtained 12 months after frame removal. Forty-six patients completed the assessment 12 months after frame removal (77\%). The mean age of the patient at the time of fracture was 54.6 years (range 31-86). There were 19 males and 27 females. At 12 months after frame removal, the mean EQ5D-5L index was 0.66 (CI 0.60-0.72). The mean EQ5D-5L VAS was 69 (CI 61-76). When this was compared to the established reference population from Denmark, the study population showed a significantly worse EQ5D-5L index. The majority of patients (87\%) were in the lower social classes suggesting a higher degree of social deprivation in the study population. Twenty-seven per cent of patients who were employed prior to injury had returned to employment at approximately
\end{abstract}

Rasmus Elsoe

rasmus.elsoe@gmail.com

1 Department of Orthopaedic Surgery, Aalborg University Hospital, Aalborg University, 18-22 Hobrovej, 9000 Aalborg, Denmark

2 Department of Occupational Therapy and Physiotherapy, Aalborg University Hospital, Aalborg, Denmark
19 months following fracture. The onset of post-traumatic osteoarthritis was present in the knee joint in $29 \%$ of patients following a proximal intra-articular fracture, whereas osteoarthritis was present at the ankle joint in $35 \%$ of patients following a distal intra-articular fracture 12 months after frame removal. This study indicates that at 12 months after frame removal there are poorer patient-reported QOL as when compared to reference populations. Furthermore, this study suggests that complex tibial fractures are associated with lower social classes and that only $27 \%$ of patients in this sample, who prior to injury were employed, had returned to employment at approximately 19 months after the injury.

Keywords Ilizarov $\cdot$ Ring fixator $\cdot$ Complex fracture tibial bone $\cdot$ Plateau fracture $\cdot$ Pilon fracture $\cdot$ Outcome

\section{Introduction}

The treatment of complex fractures of the tibia, which is defined as involving either the knee or ankle joint surfaces, multi-fragmented shaft fractures or those with major soft tissue damage, is challenging [1,2]. The long-term outcomes are associated with a risk of knee pain, malalignment, persistent articular damage and an increased risk of post-traumatic osteoarthritis [1, 3-13].

The operative treatment of such injuries is difficult due to the presence of multiple fragments. For joint injuries, the objective of treatment is anatomical reduction, restoration of axial alignment and stable fixation until union $[1,2]$. The literature describes several treatment methods-involving external fixation, staged treatment and internal fixation-all with mixed results [1, 3-13]. A number of reports have reported favourable results of the treatment with a ring fixator $[3,4,10,12-15]$, and this is 
the preferred method of the authors. Prospective studies evaluating patient-reported outcomes, function and development of osteoarthritis following such fractures treated with ring external fixation are lacking.

Axial malalignment and articular injuries of the knee and ankle may increase the risk of post-traumatic osteoarthritis which, in turn, leads to a total knee replacement or fusion of the ankle joint at an early age [7]. Consequently, complex articular fractures of the tibia may produce a significant socio-economic influence on the patient group and lead to a decreased quality of life (QOL) [7].

The primary aim of this study was to report the patientreported health-related quality of life (QOL) at 12 months after frame removal following a complex fracture of the tibia. The secondary aim was to describe the socio-economic characteristics of the patient sample and report the rate of return to work 12 months after frame removal.

The hypothesis was that patients would report a worse outcome compared with the Danish reference population on EQ5D-5L index score at 12 months after frame removal after a complex tibial fracture.

\section{Patients and methods}

\section{Study design}

This was a prospective study of all patients with a complex tibial fracture treated with a ring external fixator. This study reports the outcomes 12 months after frame removal. The Danish Data Protection Agency (J. nr. 200858-0028) approved the study.

The primary outcome measurement was the EQ5D$5 \mathrm{~L}$ index [16]. The secondary outcomes were patientreported questionnaires (KOOS, OMAS, MDI), radiological outcomes and the sample's social class and employment status.

All patients were treated with a ring external fixator for a complex fracture of the tibia at Aalborg University Hospital, Denmark, between December 2012 and May 2014. These patients were included in the Trauma Ilizarov Database. Patients with complex tibial fractures not treated with a ring external fixator were excluded. Patients who were unable to fill out the questionnaires due to physical or mental disabilities were excluded. A detailed overview of patient demographics is shown in Fig. 1.

Information about age, gender, fracture classification, co-morbidities and socio-economic data was registered. Fracture classification was performed according to the AO classification [17] and was conducted on the preoperatively obtained CT scan.

\section{Surgical treatment}

Bicondylar fractures of the tibial bone, complex fractures with soft tissue damage of the tibial shaft and distal fractures of the tibial bone not treatable safely by intramedullary nailing were all treated by a ring external fixator. The authors preferred to manage proximal and distal intra-articular tibial fractures with initial screw fixation of articular bone fragments and, if necessary, with exposure of the joint surface. Both autogenous bone grafting and allogenic bone grafting were used. The metaphyseal-diaphyseal junctions of the fracture were bridged by the circular frame. The frame was attached to the bone by hydroxyapatite-coated half pins and Kirschner wires with olives. After applying the ring, external fixator alignment was assessed and corrected. Amendments such as footplates and proximal fixation of the femur were used when deemed appropriate.

\section{Outcome measurements 12 months after frame removal}

\section{Patient-reported measurements}

EQ5D-5L is a standardized and validated instrument to assess health outcome [16]. It consists of five dimensions: mobility, self-care, usual activities, pain or discomfort, anxiety or depression, and a self-rated health scale on a $20-\mathrm{cm}$ vertical, visual analogue scale with endpoints labelled 'the best health you can imagine' and 'the worst health you can imagine'. Each dimension has five levels: no problems, slight problems, moderate problems, severe problems and extreme problems. A Danish data set was used to calculate the EQ5D-5L index [18]. An EQ5D-5L index of 1.0 indicates full health and 0.0 denotes death. A reference population from Denmark is available [19].

The Knee Injury and Osteoarthritis Outcome Score (KOOS) [20] is a standardized and validated instrument used to evaluate knees and associated problems. The questionnaire includes 42 items, and each item obtains a score from 0 to 4; a total score from 0 to 100 is calculated for each subscale. A total score of 100 indicates no symptoms and 0 indicates major symptoms. KOOS reference data [21] from a general population-based sample in southern Sweden are available.

The Olerud-Molander Ankle Score (OMAS) [22] is a standardized and validated instrument used to evaluate ankle and associated problems. The OMAS is a patient-reported questionnaire, developed to evaluate function after ankle fracture. The scale is a functional rating scale from 0 (totally impaired) to 100 (completely unimpaired) and is based on nine different items: pain, stiffness, swelling, stair climbing, running, jumping, squatting, supports and activities of daily living. 


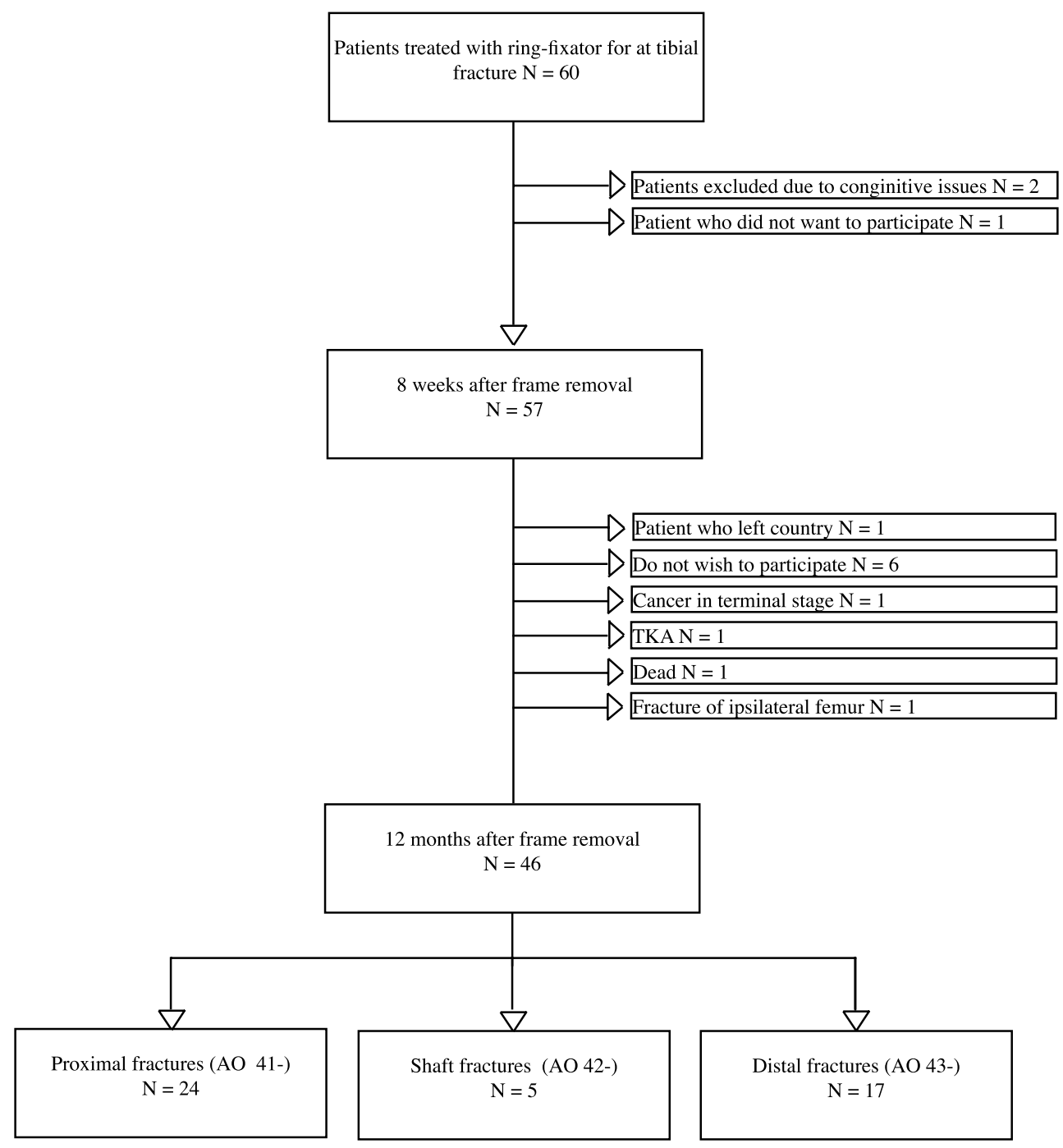

Fig. 1 Detailed overview of the study population

The Major Depression Inventory (MDI) score [23] is a validated system designed to measure depression symptoms in accordance with the symptom guidelines defined by the WHO classification for unipolar depression (ICD10) and the American Psychiatric Association classification for major depression (DSM-IV). The instrument consists of 12 questions. On a six-point Likert scale, the individual items measure how much of the time the symptoms have been present during the last 14 days. The MDI was scored according to specific guidelines. A score of zero indicates no depression and 50, severe depression. The following categories were used: no depression, less than 20, mild, 20-24, moderate, 25-29 and severe depression, 30 or more, were used [23, 24].

Pain was assessed on a visual analogue scale (VAS) ranging from 0 to $10 \mathrm{~cm}$. Patients were asked to classify pain while resting.

\section{Socio-economic evaluation and return to work}

The patients' social class was recorded based on a method developed by the Danish National Institute of Social Research [25]. The patients were grouped in five social classes according to three criteria: occupation, education and number of subordinates. The classes were defined as follows: (1) consists of university graduates, self-employed individuals with more than 20 employees, and salaried employees with more than 50 subordinates, (2) consists of self-employed individuals with 6-20 employees and salaried employees with 11-50 subordinates or a medium-long education, (3) consists of self-employed individuals with a minimum of five employees and salaried employees with 1-10 subordinates or with specialized work, (4) includes salaried employees and lower-level and skilled manual workers and (5) consists of unskilled manual workers [25]. 
Socio-economic evaluation is presented with a comparison of the patient's pre-injury employment status and employment status 12 months after frame removal. The outcomes included return to work defined as: (1) returned to pre-injury work, (2) returned to work with reduced time, (3) not able to return to work and (4) retired before injury. Data were collected through an interview at the time of admission to the hospital and at follow-up 12 months after frame removal.

\section{Radiological outcome measurements}

Radiographic examination, including X-rays and preoperative CT scans were obtained from all patients. Post-operatively, X-rays of the entire lower leg were obtained and used to evaluate the quality of reduction. Radiological examination was performed 12 months after frame removal. Intraarticular fractures of the proximal tibia were evaluated for alignment, residual depression of the articular surface and condylar widening as described by Rasmussen et al. [26]. Shaft fractures were evaluated for alignment. Distal intraarticular fractures were evaluated for alignment, talar subluxation, residual central depression and mortise widening as described by Ramos et al. [4]. Furthermore, an assessment of the quality of reduction in distal fractures was performed as described by Marsh et al. [27], modified by Burwell and Charnley [28]. Osteoarthritis was evaluated as described by Kellgren and Lawrence [29]. X-rays were obtained nonweight bearing 6 weeks post-operatively and with weight bearing at follow-up 12 months after frame removal. Radiological outcomes were assessed by a single author (RE).

The authors have evaluated the patient-reported satisfaction during the treatment period of ring external fixation in this same patient group previously. This study presents an extension of that study on the same sample.

\section{Statistics}

The distribution of variables was checked visually for normality by QQ plots. Continuous data were expressed as mean and standard deviation (SD). Categorical data were expressed as frequencies. The statistical analysis was performed by Stata (version 13).

\section{Results}

Sixty patients were treated with a ring external fixator for a complex fracture of the tibia during the study period. A total of 46 patients completed the examination 12 months after frame removal (77\%) and were included in the present study. All fractures united during the study period. Fourteen patients were lost to follow-up. A detailed overview of the patients entering the study is presented in Fig. 1.

Twenty-four patients were treated after a proximal tibial plateau fracture, five patients following a diaphyseal fracture and 17 patients following an intra-articular distal tibial fracture. The mean age at the time of fracture was 54.6 years, with a range of 31-86 years. Baseline variables of all patients are presented in Table 1.

\section{Patient-reported quality of life}

Twelve months after frame removal, the mean EQ5D-5L index was 0.66 (SD 0.19). The mean EQ5D-5L VAS was 69 (SD 24.4). Compared with the established reference population from Denmark [19], the study population showed a significantly worse EQ5D-5L index. The EQ5D-5L scores divided into AO type 41, 42 and 43 are presented in Table 2.

Table 1 Baseline characteristics

\begin{tabular}{|c|c|}
\hline Follow-up time from injury, months (SD) & $19.3(3.3)$ \\
\hline Follow-up time from frame removal, months (SD) & $12.7(3.3)$ \\
\hline Age at time of fracture, mean (range) & $54.6(31-86)$ \\
\hline Gender male/female & $19 / 27$ \\
\hline BMI, mean (SD) & $25.6(5.1)$ \\
\hline Smoker yes/no & $28 / 18$ \\
\hline Side of injury, right/left/bilateral & $22 / 22 / 2$ \\
\hline High-/low-energy trauma & $16 / 30$ \\
\hline Poly-/mono-trauma & $11 / 35$ \\
\hline \multicolumn{2}{|l|}{ Co-morbidities } \\
\hline ASA score, mean (SD) & $1.7(0.6)$ \\
\hline Charlston co-morbidity score, mean (SD) & $2.8(1.7)$ \\
\hline Diabetes mellitus & 6 \\
\hline \multicolumn{2}{|l|}{ Fracture classification $(A O)$} \\
\hline AO-41 & 24 \\
\hline A & 2 \\
\hline $\mathrm{B}$ & 0 \\
\hline $\mathrm{C}$ & 22 \\
\hline $\mathrm{AO}-42$ & 5 \\
\hline A & 3 \\
\hline $\mathrm{B}$ & 0 \\
\hline $\mathrm{C}$ & 2 \\
\hline AO-43 & 17 \\
\hline A & 5 \\
\hline B & 6 \\
\hline $\mathrm{C}$ & 6 \\
\hline Open/closed fracture & $4 / 42$ \\
\hline AO-41 & 1 \\
\hline $\mathrm{AO}-42$ & 1 \\
\hline $\mathrm{AO}-43$ & 2 \\
\hline
\end{tabular}

AO classification [17]

$S D$ standard deviation 
Table 2 Patient-reported outcomes 12 months after frame removal compared with reference populations

\begin{tabular}{|c|c|c|c|c|c|}
\hline & \multicolumn{5}{|l|}{ KOOS } \\
\hline & PAIN & ADL & SYMP & QOL & SPORT \\
\hline \multicolumn{6}{|c|}{ Proximal fracture ( $\mathrm{AO}$ 41-) } \\
\hline \multicolumn{6}{|c|}{ Study population } \\
\hline Mean & 68.9 & 71.4 & 64.9 & 52.1 & 30.4 \\
\hline $95 \% \mathrm{CI}$ & $58.5-79.2 *$ & $62.2-80.7 *$ & $54.3-75.4^{*}$ & $39.0-65.3^{*}$ & $19.2-41.6^{*}$ \\
\hline \multicolumn{6}{|c|}{ Reference population $* * * * *$} \\
\hline \multirow[t]{3}{*}{$95 \% \mathrm{CI}$} & $86.7-88.2$ & $86.5-88.1$ & $85.4-86.9$ & $77.4-79.6$ & $72.5-75.1$ \\
\hline & & & \multicolumn{3}{|l|}{ EQ5D-5L } \\
\hline & & & Index & & VAS \\
\hline \multicolumn{6}{|c|}{ Study population } \\
\hline Mean & & & 0.715 & & 76 \\
\hline $95 \% \mathrm{CI}$ & & & $0.635-0.795 *$ & & $67.5-84.4$ \\
\hline \multicolumn{6}{|c|}{ Reference population $* * * *$ (male/female $50-59$ years) } \\
\hline Mean & & & $0.888 / 0.858$ & & \\
\hline \multirow[t]{3}{*}{$95 \% \mathrm{CI}$} & & & $0.880-0.896 / 0.850-0.866$ & & \\
\hline & & & \multicolumn{3}{|l|}{ EQ5D-5L } \\
\hline & & & Index & & VAS \\
\hline \multicolumn{6}{|c|}{ Shaft fracture ( $A O$ 42-) } \\
\hline \multicolumn{6}{|c|}{ Study population } \\
\hline Mean & & & 0.681 & & 56 \\
\hline $95 \% \mathrm{CI}$ & & & $0.576-0.786^{*}$ & & $27.8-84.2$ \\
\hline \multicolumn{6}{|c|}{ Reference population $* * * *$ (male/female $50-59$ years) } \\
\hline Mean & & & $0.888 / 0.858$ & & \\
\hline \multirow[t]{3}{*}{$95 \% \mathrm{CI}$} & & & $0.880-0.896 / 0.850-0.866$ & & \\
\hline & & & \multicolumn{3}{|l|}{ EQ5D-5L } \\
\hline & & & Index & & VAS \\
\hline \multicolumn{6}{|c|}{ Distal fracture (AO 43-) } \\
\hline \multicolumn{6}{|c|}{ Study population } \\
\hline Mean & & & 0.591 & & 63.7 \\
\hline $95 \% \mathrm{CI}$ & & & $0.476-0.706^{*}$ & & $48.5-79.0$ \\
\hline \multicolumn{6}{|c|}{ Reference population $* * * *$ (male/female $50-59$ years) } \\
\hline Mean & & & $0.888 / 0.858$ & & \\
\hline $95 \% \mathrm{CI}$ & & & $0.880-0.896 / 0.850-0.866$ & & \\
\hline
\end{tabular}

* Significantly different compared with reference population

** Paradowski et al. [21]

*** Unpublished data. Ewa Roos 'Personal communication' 13 November 2012. Paradowski et al. [21]

**** Sorensen et al. [19]

\section{Socio-economic evaluation and return to work}

The distribution of social classes at the time of admission to hospital showed three patients in group I, three patients in group III, 22 patients in group IV and 18 patients in group $\mathrm{V}$. The majority (87\%) of patients were grouped into social classes IV and V, indicating a high degree of social deprivation in the study population.
Thirty-four of the 46 patients in the study population were below the age of 65 years, which was the official retirement age in Denmark in 2014. Twenty of these 34 patients were employed prior to the occurrence of the injury, and two patients above the age of 65 years were employed. Of the 22 patients who were employed prior to the injury, six patients returned to pre-injury work, nine patients were employed with reduced working hours, and seven patients were unable 
to return to work, 12 months after frame removal. Both patients above the age of 65 years returned to employment.

\section{Indication of depression}

Twelve months after frame removal, the MDI score, indicating psychological status, showed 41 patients reporting no depression. Four patients reported MDI scores between 20 and 30, indicating mild-to-moderate depression, and one patient reported a score of more than 30 , indicating severe depression.

\section{Tibial plateau fracture (AO 41-): 24 patients}

Twelve months after frame removal, the mean KOOS score were pain, 69 (SD 24); symptoms, 65 (SD 26); ADL, 71 (SD 24); sport, 30 (SD 29); and QOL 52 (SD 32). Compared with the established reference population [21], the study population showed a significantly worse KOOS outcome for all five subgroups. See Table 2.

The VAS score for resting pain was reported as a range from zero to six with an average of 1.3 (SD 2.1). Sixteen patients reported no pain at rest, five patients reported a VAS between one and three, and three patients reported a VAS scores between four and six.

\section{Radiological outcomes}

Five of the 24 patients had either malalignment, condylar widening more than $5 \mathrm{~mm}$ and/or articular depression of more than $5 \mathrm{~mm} 12$ months after frame removal. A detailed overview is presented in Table 3 . The radiological outcomes of osteoarthritis of the knee (Kellgren and Lawrence) show three patients with no or doubtful signs of osteoarthritis (Type 0 and Type 1), 13 patients with minimal signs of osteoarthritis (Type 2) and six patients with moderate signs of osteoarthritis (Type 3). One patient with Type 3 osteoarthritis was treated with a total knee arthroplasty (TKA) during the study period.

\section{Tibial diaphyseal fracture (AO-42): five patients}

The VAS score for resting pain was reported as a range from 0 to 7 with an average of 1.4 (SD 3.1). Four patients reported no pain at rest and one patient reported a VAS score of seven.

\section{Radiological outcomes}

One of the five patients treated for a shaft fracture had a varus deformity of six degrees. The radiological outcomes
Table 3 Radiological malalignment

\begin{tabular}{ll}
\hline Proximal (41) & \\
Malalignment $>3^{\circ}$ & 2 \\
Condylar widening $>5 \mathrm{~mm}$ & 2 \\
Depression $>5 \mathrm{~mm}$ & 3 \\
Number of affected patients & 5 \\
Shaft (42) & 1 \\
Malalignment $>3^{\circ}$ & \\
Distal (43) & 1 \\
Malalignment $>3^{\circ}$ & 2 \\
Central Depression $>5 \mathrm{~mm}$ & 3 \\
Number of affected patients & \\
\hline
\end{tabular}

12 months after frame removal, the radiological assessments were made on AP and side X-rays. Proximal tibial fractures were evaluated concerning alignment and depression of the articular surface and condylar widening as described by Rasmussen et al. [27]. Shaft fractures were evaluated concerning alignment. Distal fractures were evaluated with regard to alignment, talar subluxation, central depression and mortise widening as described by Ramos et al. [4]

for osteoarthritis of the ankle and knee (Kellgren and Lawrence) showed five patients with none or doubtful signs of osteoarthritis (Type 0 and 1 ).

\section{Fracture of the distal tibia (AO 43-): 17 patients}

The mean Olerud-Molander Ankle Score 12 months after frame removal was 43(SD 32). No reference population was available for the Olerud-Molander Ankle Score.

The VAS score for resting pain was reported as a range from zero to eight with an average of 1.0 (SD 2.4). Thirteen patients reported no pain at rest, two patients reported a VAS between one and three, one patients reported a VAS of five, and one patient reported a VAS score of eight.

\section{Radiological outcomes}

Three of the 17 patients treated for a distal intra-articular fracture had either malalignment of more than 3 degrees or a central depression of more than $5 \mathrm{~mm}$. A detailed overview is presented in Table 3. The radiological outcomes of osteoarthritis of the ankle (Kellgren and Lawrence) showed seven patients with no or doubtful signs of osteoarthritis (Type 0 and 1), four patients with minimal signs of osteoarthritis (Type 2), four patients with moderate signs of osteoarthritis (Type 3 ) and two patients with severe signs of osteoarthritis (Type 4).

These two patients with Type 4 osteoarthritis were treated with a talocrural fusion (11 and 32 months following primary injury, respectively). 


\section{Discussion}

The treatment of these fractures of the tibia is challenging due to the multi-fragmented nature and the involvement of joint surfaces and concomitant soft tissue injuries. Moreover, the fractures are often a result of high-energy trauma [3, 4] and significant complications during treatment are common [2, 30, 31].

This study shows a considerable social deprivation of the study population with $87 \%$ of the population in the lower social classes (IV and V) compared with the general population in Denmark with $46 \%$ in the lower social classes [32]. This increased incidence of complex tibial fractures in the lower social classes with an increased likelihood of deprivation is supported by Court-Brown et al. [33] who reported a significant increase in the incidence of fractures in the most deprived $10 \%$ of the population with most fracture types affected. Whether this increased risk is based on behavioural differences, is work related or is due to economic differences or other factors, is not well established.

Seventy-four per cent of the study population was below the retirement age of 65 years. Fifty-nine per cent of the patients below 65 years of age were employed prior to injury. There was a substantially higher degree of unemployment in the study group compared with the national Danish general unemployment rate of less than 5\% [34]. Twelve months after frame removal, $27 \%$ of the patients who had been employed prior to the fracture had returned to work. This suggests a high risk of attrition from employment in the study population after a complex fracture of the tibia. This might be due to the high proportion of lower social classes in the sample which are often employed in manual labour. This type of employment might indicate an increased dependency on a high level of function of the lower extremity. Furthermore, as part of the Danish flexicurity model, Denmark has a high level of income benefit constituting upwards of $90 \%$ [40] of the lowest salaries initially, and this may influence the rate of employment of those with disability.

Taking individual patient information into account when planning post-operative treatment methods may be important. Development of special rehabilitation algorithms directed towards this patient group including social and work-related interventions may improve outcome. However, more research is needed to combine social and health science.

The patient-reported outcomes 12 months after frame removal showed results below the established reference populations $[19,21]$ in both generic (EQ5D-5L) and symptom-specific questionnaires (KOOS). Studies reporting on patient-reported outcome following complex fractures of the tibia support the persistence of limitations in QOL throughout time $[3,4,15,35]$. However, most studies evaluating patient-reported outcomes do not use a reference population or pre-injury values. A recent study by The Canadian Orthopaedic Trauma Society [15] indicated that patient-reported outcomes are more sensitive to residual disability than radiological evaluations. Furthermore, it is documented that low levels of education are associated with a worse QOL [19].

The onset of post-traumatic osteoarthritis following fractures of the tibia involving the joint surfaces has been the subject of several studies. The incidence of osteoarthritis following tibial condyle fractures is reported to be between 17 and $83 \%$ with a wide range in the severity of osteoarthritis $[1,36]$. In the present study, $29 \%$ of patients with proximal tibial plateau fractures demonstrated moderate-to-severe osteoarthritis of the knee at a mean of 19.3 months follow-up after fracture. These findings are in line with other studies reporting osteoarthritis following tibial plateau fractures $[7,15]$.

Moreover, early onset of osteoarthritis is reported in distal fractures involving the ankle joint $[4,37]$. In the present study, $35 \%$ of the patients in the group with distal fractures demonstrated moderate-to-severe signs of osteoarthritis at follow-up, which is comparable to previous studies reporting on osteoarthritis following distal tibial fractures [37].

This study has several limitations. First, this is a prospective follow-up study which precludes the drawing of conclusions regarding causality. Another limitation of this study is the number of patients included, which limits comparisons or other associations between the groups. Furthermore, the study is limited by a minimum follow-up time of 12 months after frame removal. Other studies have shown that the development of post-traumatic osteoarthritis is affected by the time after the fracture [38, 39]. Regarding the social classes and return to previous employment, this study evaluates patients in a country with a high degree of social insurance including a high level of publicly financed unemployment insurance. This may lead to a lower level of incentive to return to work as compared with other countries.

\section{Conclusion}

This study shows that patients 12 months after frame removal report significantly worse patient-reported QOL as compared to reference populations. Furthermore, this study demonstrates that complex tibial fractures are associated with lower social classes and that $27 \%$ of patients, who prior to injury were employed, returned to employment at approximately 19 months after the fracture.

Acknowledgements The Department of Orthopaedic Surgery and the Department of Occupational and Physiotherapy, Aalborg University Hospital, Denmark, is acknowledged for proving unrestricted grants. 
Funding The authors did not receive benefits or grants in any form from a commercial part related directly or indirectly to the subject of this article.

\section{Compliance with ethical standards}

Conflict of interest The authors declare no conflict of interest. No financial support was received for this study.

Ethical standards All procedures performed in the study were in accordance with the ethical standards of the regional national research committee and with the 1964 Declaration of Helsinki and its later amendments or comparable ethical standards.

Informed consent Informed consent was obtained from individual participants included in the study.

Open Access This article is distributed under the terms of the Creative Commons Attribution 4.0 International License (http://creativecommons.org/licenses/by/4.0/), which permits unrestricted use, distribution, and reproduction in any medium, provided you give appropriate credit to the original author(s) and the source, provide a link to the Creative Commons license, and indicate if changes were made.

\section{References}

1. Jansen H, Frey SP, Doht S et al (2013) Medium-term results after complex intra-articular fractures. J Orthop Sci 8(4):569-577

2. Joveniaux P, Ohl X, Harisboure A et al (2010) Distal tibia fractures: management and complications of 101 cases. Int Orthop 34:583-588

3. Ramos T, Ekholm C, Eriksson BI et al (2013) The Ilizarov external fixator-a useful alternative for the treatment of proximal tibial fractures. A prospective observational study of 30 consecutive patients. BMC Musculoskelet Disord 14:11

4. Ramos T, Karlsson J, Eriksson BI, Nistor L (2013) Treatment of distal tibial fractures with the Ilizarov external fixator-a prospective observational study in 39 consecutive patients. BMC Musculoskelet Disord 14:30

5. Krappinger D, Irenberger A, Zegg M, Huber B (2013) Treatment of large posttraumatic tibial bone defects using the Ilizarov method: a subjective outcome assessment. Arch Orthop Trauma Surg 133:789-795

6. Ali AM (2013) Outcomes of open bicondylar tibial plateau fractures treated with Ilizarov external fixator with or without minimal internal fixation. Eur J Orthop Surg Traumatol 23:349-355

7. Manidakis N, Dosani A, Dimitriou R et al (2010) Tibial plateau fractures: functional outcome and incidence of osteoarthritis in 125 cases. Int Orthop 34:565-570

8. Sament R, Mayanger JC, Tripathy SK, Sen RK (2012) Closed reduction and percutaneous screw fixation for tibial plateau fractures. J Orthop Surg (Hong Kong) 20:37-41

9. Subasi M, Kapukaya A, Arslan H et al (2007) Outcome of open comminuted tibial plateau fractures treated using an external fixator. J Orthop Sci 12:347-353

10. Korkmaz A, Ciftdemir M, Ozcan M et al (2013) The analysis of the variables, affecting outcome in surgically treated tibia pilon fractured patients. Injury 44:1270-1274

11. Ahearn N, Oppy A, Halliday R et al (2014) The outcome following fixation of bicondylar tibial plateau fractures. Bone Joint $\mathrm{J}$ 96-B:956-962
12. Metcalfe D, Hickson CJ, McKee L, Griffin XL (2015) External versus internal fixation for bicondylar tibial plateau fractures: systematic review and meta-analysis. J Orthop Traumatol 16(4):275-285

13. Dall'oca C, Maluta T, Lavini F et al (2012) Tibial plateau fractures: compared outcomes between ARIF and ORIF. Strateg Trauma Limb Reconstr 7:163-175

14. Ali AM, Burton M, Hashmi M, Saleh M (2003) Outcome of complex fractures of the tibial plateau treated with a beamloading ring fixation system. J Bone Joint Surg Br 85:691-699

15. Canadian Orthopaedic Trauma Society (2006) Open reduction and internal fixation compared with circular fixator application for bicondylar tibial plateau fractures. Results of a multicenter, prospective, randomized clinical trial. J Bone Joint Surg Am 88:2613-2623

16. Eq-5d questionnary (2012) http://www.euroqol.org/about-eq-5d/ publications/user-guide.html

17. Marsh JL, Slongo TF, Agel J et al (2007) Fracture and dislocation classification compendium-2007: Orthopaedic Trauma Association Classification, database and outcome committee. J Orthop Trauma 21(10 Suppl):S1-S133

18. Wittrup-Jensen KU, Lauridsen J, Gudex C, Pedersen KM (2009) Generation of a Danish TTO value set for EQ-5D health states. Scand J Public Health 37:459-466

19. Sorensen J, Davidsen M, Gudex C et al (2009) Danish EQ-5D population norms. Scand J Public Health 37:467-474

20. KOOS questionnaire [KOOS web site]. http://www.koos.nu. Accessed 12 June 2011

21. Paradowski PT, Bergman S, Sunden-Lundius A et al (2006) Knee complaints vary with age and gender in the adult population. Population-based reference data for the Knee injury and Osteoarthritis Outcome Score (KOOS). BMC Musculoskelet Disord 7:38

22. Olerud C, Molander H (1984) A scoring scale for symptom evaluation after ankle fracture. Arch Orthop Trauma Surg 103:190-194

23. Olsen LR, Jensen DV, Noerholm V et al (2003) The internal and external validity of the Major Depression Inventory in measuring severity of depressive states. Psychol Med 33:351-356

24. Bech P, Rasmussen NA, Olsen LR et al (2001) The sensitivity and specificity of the Major Depression Inventory, using the Present State Examination as the index of diagnostic validity. J Affect Disord 66:159-164

25. Moller L, Kristensen TS, Hollnagel H (1991) Social class and cardiovascular risk factors in Danish men. Scand J Soc Med 19:116-126

26. Rasmussen PS (1973) Tibial condylar fractures. Impairment of knee joint stability as an indication for surgical treatment. $\mathrm{J}$ Bone Joint Surg Am 55:1331-1350

27. Marsh JL, Buckwalter J, Gelberman R et al (2002) Articular fractures: does an anatomic reduction really change the result? J Bone Joint Surg Am 84-A:1259-1271

28. Burwell HN, Charnley AD (1965) The treatment of displaced fractures at the ankle by rigid internal fixation and early joint movement. J Bone Joint Surg Br 47:634-660

29. Kellgren JH, Lawrence JS (1957) Radiological assessment of osteo-arthrosis. Ann Rheum Dis 16:494-502

30. McFerran MA, Smith SW, Boulas HJ, Schwartz HS (1992) Complications encountered in the treatment of pilon fractures. J Orthop Trauma 6:195-200

31. Papagelopoulos PJ, Partsinevelos AA, Themistocleous GS et al (2006) Complications after tibia plateau fracture surgery. Injury 37:475-484

32. Olsen L, Ploug N, Andersen L, Juul JS (2012) Det Danske Klassesamfund (the Danish Class Society), 1st edn. Gyldendal, Copenhagen 
33. Court-Brown CM, Aitken SA, Duckworth AD et al (2013) The relationship between social deprivation and the incidence of adult fractures. J Bone Joint Surg Am 95:e321-e327

34. Danmarks Statistik. http://www.dst.dk/da/Statistik/ NytHtml?cid=19416. 18 Oct 2015

35. Jacob N, Amin A, Giotakis N et al (2015) Management of highenergy tibial pilon fractures. Strateg Trauma Limb Reconstr 10(3):137-147

36. Rademakers MV, Kerkhoffs GM, Sierevelt IN et al (2007) Operative treatment of 109 tibial plateau fractures: five- to 27-year follow-up results. J Orthop Trauma 21:5-10
37. Abd-Almageed E, Marwan Y, Esmaeel A et al (2015) Hybrid external fixation for Arbeitsgemeinschaft fur Osteosynthesefragen (AO) 43-C tibial plafond fractures. J Foot Ankle Surg 54(6):1031-1036

38. Houben PF, van der Linden ES, van den Wildenberg FA, Stapert JW (1997) Functional and radiological outcome after intra-articular tibial plateau fractures. Injury 28:459-462

39. Honkonen SE (1995) Degenerative arthritis after tibial plateau fractures. J Orthop Trauma 9:273-277

40. HK. Danish flexicurity model. https://www.hk.dk/akasse/emner/ dagpengesatser. Published 2017. Accessed March 2017 